\title{
Conductimetric Titrations of Acids and Bases in Benzene and Dioxane
}

\author{
By Arthur A. Maryott
}

\begin{abstract}
Acid-base titrations were made conductimetrically in pure benzene and dioxane with picric, trichloroacetic, and camphorsulfonic acids, together with primary, secondary, and tertiary amines. Although the conductances of the solutions were extremely low, lower by a factor of $10^{-8}$ or more than in water, the titrations gave sharp end points which generally were accurate to 1 percent or better. The unusual, though similar behavior of all titrations involving trichloroacetic or camphorsulfonic acid, where the conductance of the salt was enhanced greatly by the presence of free acid, was interpreted in terms of a reaction between salt and acid leading to the formation of a complex anion. The occasional variations in conductance, with time, in one instance suggesting slow attainment of some secondary ionic equilibrium, and the effect upon the titration curves of the addition of a small amount of methyl alcohol to the solvent are discussed.
\end{abstract}

\section{Introduction}

While the conductimetric method has been employed as a general procedure for acid-base titrations in water and has been extended to certain nonaqueous and mixed solvents, its application to purely nonpolar solvents such as benzene and dioxane which have very low dielectric constants remains practically an unexplored field. One case has been reported by La Mer and Downes [1] ${ }^{1}$ in which trichloroacetic acid was titrated with diethylamine in benzene. The titration curve shown by these workers exhibited an interesting and rather unexpected behavior. On titration of the acid with the base, the conductance first rose to a maximum well before the equivalence point was reached and then decreased to a minimum somewhere in the vicinity of the equivalence point. It was not apparent from their data, however, whether there was any definite break in the titration curve at the equivalence point which would be suitable for analytical purposes. The present work was undertaken not only to explore the possibility of extending an analytical method, but also in the hope that additional information concerning the behavior of electrolytes in these media might be obtained.

\footnotetext{
1 Figures in brackets indicate the literature references at the end of this
} paper.
In solvents like benzene which serve primarily as inert diluents, dilute solutions of acids or bases have almost negligibly low conductances. There is practically no tendency for the solvent to react with the acid or base to form ions as would be the case in an amphoteric solvent like water. When the solution contains both acid and base, however, they combine with the transfer of a proton from the acid to the base ${ }^{2}$ to form a true salt. Since the solvent has a low dielectric constant and has little tendency to stabilize individual ions through solvation, the extent of dissociation of the salt into free, or conducting, ions is quite small. The behavior of certain types of organic salts, particularly the tertiary and quarternary ammonium halides and picrates, have been extensively investigated by Walden [2] and by Kraus [3] and coworkers. The very low conductances of these solutions indicate that only a minute fraction of the salt, something of the order of a millionth of 1 percent, is dissociated into free ions. The remainder exists as nonconducting ion pairs, or aggregates of jon pairs, held together largely by coulomb torces. Although the equivalent con-

\footnotetext{
2 The definition of acid employed here is that of Brönsted rather than the more generalized electronic concept of Lewis. Although compounds owing their acidity to an electron deficiency can be titrated with the aid of colored indicators, these acids are not suitable for the present purpose since an ionic product having a measurable conductance is required.
} 
ductance varies in a rather complex manner with concentration, and suggests a multiple series of equilibria involving the formation of triple ions and more highly associated ionic products, the specific conductance increases continually with concentration. Conductimetric titrations in these solvents might be expected to follow a simple pattern. The extent of ionization of the salt, though small, should be considerably greater than that of either the acid or the base, so that the conductance would be determined almost entirely by the concentration of salt formed. Then the conductance would be expected to increase more or less uniformly until the equivalence point is reached, and remain substantially constant upon the addition of excess titrating solution. It is evident from the experimental results that only a part of the titrations conform to this simple pattern.

The choice of acids and bases is limited by the solubility, particularly of the salt formed, and by the requirement that they be sufficiently strong to insure complete neutralization. In these respects picric and trichloroacetic acids together with various aliphatic primary, secondary, and tertiary amines were for the most part suitable. Titrations were generally made in both dioxane and benzene. To determine the effect of a small amount of polar impurity, in some cases approximately 1 percent of methyl alcohol was also added to the solvent before titrating. Camphorsulfonic acid was used in several titrations in dioxane. Good breaks in the titration curves, corresponding to the equivalence points calculated from aqueous standardizations of the various solutions, were observed in practically all cases.

\section{Materials}

Reagent-grade trichloroacetic acid was stored in a vacuum desiccator until used. Picric acid and $d l$-camphor-sulfonic acid were recrystallized, respectively, from benzene and dioxane, and dried in a vacuum oven. The various amines, obtained from Eastman Kodak Co., and Sharples Chemicals, Inc., were used without additional treatment. To remove moisture the solvents, benzene and dioxane, were refluxed and distilled over sodium.

\section{Apparatus and Procedure}

A direct-current, high-resistance bridge, General Radio Co. type 544-B, was used to measure the resistances of the cell during the titrations. These resistances were of the order of $10^{7}$ to $10^{10} \mathrm{ohms}$. Sensitivity as well as convenience and rapidity of measuring these high resistances, rather than accuracy, were determining factors in selecting the method. Throughout the required range, the resistances could be determined to 1 percent, which is as precisely as the logarithmic dial could be read. The use of direct current introduces the possibility that the measured resistances may be influenced by the effects of electrode polarization. Should these effects cause the resistance of the cell to vary appreciably with time, they might interfere with the titrations. In a majority of cases, either no such drifts were observed or else they were sufficiently small so as not to interfere with detection of the equivalence points.

A photograph of the cell and titrating assembly is shown in figure 1 . The cell consists of an inner

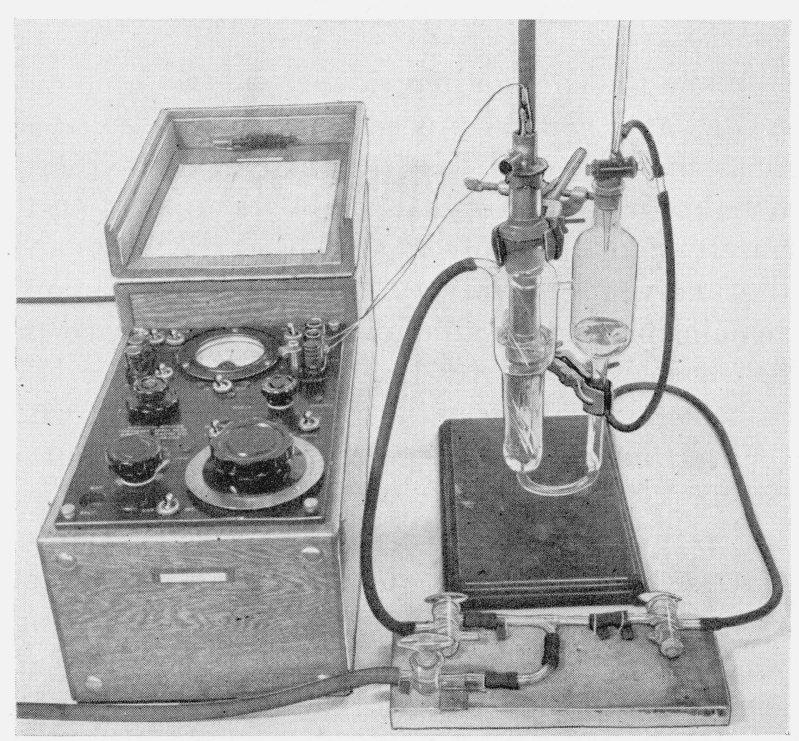

Figure 1.-Assembly used in conductimetric titratious in benzene and dioxane.

section of soft glass, on which are mounted two concentric platinum electrodes and their leads, and an outer $U$-shaped container of Pyrex glass. 
As the cell was also used in measurements of dielectric constants, the lead from the outer electrode is tubular and serves as a shield for the lead from the inner electrode. In addition to the electrode compartment, the outer section contains two enlarged bulbs to accommodate excess liquid and to facilitate mixing the solutions in the manner to be described. The electrodes are $8.0 \mathrm{~cm}$ high, with the outer one having a diameter of $2.4 \mathrm{~cm}$. The spacing between electrodes is approximately $0.2 \mathrm{~cm}$. A sufficiently good estimate of the cell constant, $k$, is obtained from the intrelectrode capacitance, $C$, by the formula, $k=d / A=1 /(4 \pi C)$. This capacitance in air, determined by calibration with pure benzene, is 27.4 $\mathrm{cm}$ so that $k=0.00291 \mathrm{~cm}^{-1}$.

Stock solutions were made up in volumetric flasks from weighed quantities of acid or base so that the concentrations were approximately 0.2 molar. To the cell containing $100 \mathrm{ml}$ of solvent was added $5 \mathrm{ml}$ of stock solution of acid (or base) from a pipette. This solution was then titrated with a stock solution of base (or acid) from a 10ml burette. The concentration of salt at the equivalence point was thus around 0.009 molar in all cases. As the titrating solution was roughly twenty times as concentrated as the solution to be titrated, the effect of dilution was minimized. Compressed air, dried by passage through a tube containing calcium chloride, was used to achieve mixing upon each addition of the titrating solution by first forcing the solution into one of the enlarged bulbs and then into the other. The burette was attached to the cell opening through a cork stopper, which also contained a tube passing to the compressed air line. With the cell virtually airtight, any danger of contaminating the solution with atmospheric moisture, which might effect the conductance during the titration, was eliminated. Very small additions of stock solution, not over three or four drops, were made near the equivalence point. Whether such small additions were necessary depended upon the shape of the titration curve. In some cases the curves were substantially linear over an appreciable region on either side of the equivalence point, while in others the marked curvature of the plots required that evaluation of the end point be based upon points within a rather limited region.
All titrations were made at $25^{\circ} \pm 0.01^{\circ} \mathrm{C}$ in an oil bath. This close control of temperature maintained constant conditions for comparative purposes, and the titrations could be made satisfactorily at ordinary room temperature. The time required to obtain a complete titration curve was about 20 minutes. Because of uncertain purity, particularly of the bases, and in order to have a check upon the reliability of the conductimetric end points, all stock solutions were standardized with aqueous solutions of sodium hydroxide and hydrochloric acid, using phenolphthalein and methyl red as indicators. Solutions of amine in benzene were added to excess acid and back titrated with alkali to eliminate loss of amine through volatilization from the benzene layer.

\section{Experimental Results}

A number of preliminary titrations were made using a variety of amines with picric acid or trichloroacetic acid in benzene. While the general shapes of the titration curves were found to be dependent upon the acid, and in some cases, upon the class of amine, no essential qualitative difference was noted within a particular class of amine. Consequently, in the titrations reported, only triethylamine, di-n-butylamine, and $n$-heptylamine representing, respectively, the tertiary, secondary, and primary classes of amines were used. Frequently, both forward and reverse titrations were made. The forward titrations are defined as those in which the base is added to the acid, and the reverse titrations as those in which the acid is added to the base.

While representative data for several of the titrations are given in table 1 , it seemed generally preferable to present the results in graphical form.

The various titration curves plotted on semi-logarithmic paper are shown in figures 2 to 6 . Figures 2 to 5 show forward titrations on the left side and reverse titrations on the right. The specific conductance is plotted on the logarithmic scale. Plotted as ab. scissa is the function $X$, defined by $X=(B-A) / D$. $A$, represents the equivalents of acid, and $B$, the equivalents of base in the solution at that particular point in the titration. $D$, is the equivalents of acid or base initially to be titrated and is thus identical with $A$ for the forward and with $B$ for 
the reverse titration. The values of $A, B$, and $D$ are based upon the concentrations of the stock solutions determined by the aqueous standardizations. Up to the equivalence point, $X$ measures the fraction of acid or base not yet neutralized, and beyond this point the fractional degree of overtitration. The forward titrations start with negative values of $X$, and the reverse titrations with positive values. The equivalence point corresponds to $X=0$.

TABLE 1.-Representative data on conductimetric titrations

\begin{tabular}{|c|c|c|c|c|c|}
\hline \multicolumn{6}{|c|}{$\begin{array}{l}\text { I. Titrations with picric acid }(0.2000 \text { molar }) \text { and di- } n \text {-butylamine } \\
(0.2013 \text { molar }) \text { in benzene }\end{array}$} \\
\hline \multicolumn{3}{|c|}{ Forward titration } & \multicolumn{3}{|c|}{ Reverse titration } \\
\hline $\begin{array}{l}\text { Base } \\
\text { added }\end{array}$ & $x$ & $\begin{array}{l}\text { Specific } \\
\text { conductance }\end{array}$ & $\begin{array}{l}\text { Acid } \\
\text { added }\end{array}$ & $x$ & $\begin{array}{l}\text { Specific } \\
\text { conductance }\end{array}$ \\
\hline $\begin{array}{c}m l \\
1.50\end{array}$ & -0.698 & $0.39 \times 10^{11}$ & $\begin{array}{c}m l \\
1.50\end{array}$ & 0.702 & $0.59 \times 10^{11}$ \\
\hline 3.00 & -.396 & 1.16 & 3.00 & .403 & 1. 40 \\
\hline 3.50 & -.296 & 1. 53 & 4.00 & .205 & 2. 06 \\
\hline 4. 00 & -.195 & 1.91 & 4. 25 & .155 & 2. 26 \\
\hline 4. 25 & -.145 & 2.12 & 4. 50 & .105 & 2. 46 \\
\hline 4. 50 & -.094 & 2. 36 & 4. 75 & .056 & 2. 64 \\
\hline 4.60 & -.074 & 2.45 & 4. 90 & .026 & 2. 74 \\
\hline 4. 70 & -.054 & 2. 53 & 4. 98 & .010 & 2. 85 \\
\hline 4. 80 & -.034 & 2. 63 & 5.09 & -.011 & 2. 91 \\
\hline 4. 90 & -.014 & 2. 72 & 5.17 & -.028 & 2. 91 \\
\hline 5.00 & .006 & 2. 78 & 5. 26 & -.045 & 2.91 \\
\hline 5.10 & .025 & 2. 78 & 5. 50 & -.092 & 2. 91 \\
\hline 5. 20 & .045 & 2. 80 & 6.00 & -.193 & 2. 88 \\
\hline 5. 30 & .066 & 2. 81 & & & \\
\hline 5. 50 & .106 & 2. 85 & & & \\
\hline 6.00 & .207 & 2. 97 & & & \\
\hline \multicolumn{6}{|c|}{$\begin{array}{l}\text { II. Titrations with trichloroacetic acid (0.1994 molar) and triethyla- } \\
\text { mine }(0.1939 \text { molar }) \text { in dioxane. }\end{array}$} \\
\hline 1.00 & -0.806 & 0.044 & 1. 00 & 0. 794 & 0.033 \\
\hline 2.00 & -.611 & .092 & 2. 00 & .588 & .036 \\
\hline 3.00 & -.416 & .114 & 3. 00 & .383 & $\ldots 049$ \\
\hline 4.00 & -.222 & .180 & 4.00 & .177 & .074 \\
\hline 4. 25 & -.172 & .180 & 4. 25 & .126 & .081 \\
\hline 4.50 & -.124 & .174 & 4. 50 & .074 & .090 \\
\hline 4. 75 & -.076 & .159 & 4. 75 & .023 & .101 \\
\hline 4. 83 & -.060 & .150 & 4.81 & .010 & .104 \\
\hline 4. 90 & -.047 & .142 & 4.87 & -.002 & .110 \\
\hline 4. 98 & -.031 & .130 & 4. 93 & -.015 & .123 \\
\hline 5.07 & -.014 & .116 & 5.00 & -.029 & .139 \\
\hline 5.15 & .002 & .109 & 5. 06 & -.040 & .161 \\
\hline 5. 23 & .017 & .108 & 5. 12 & -.054 & .178 \\
\hline 5. 50 & .070 & .107 & 5. 25 & -.080 & .197 \\
\hline \multirow[t]{2}{*}{6.00} & .167 & .107 & 5. 50 & -.131 & .285 \\
\hline & & & 6. 50 & -.337 & .415 \\
\hline
\end{tabular}

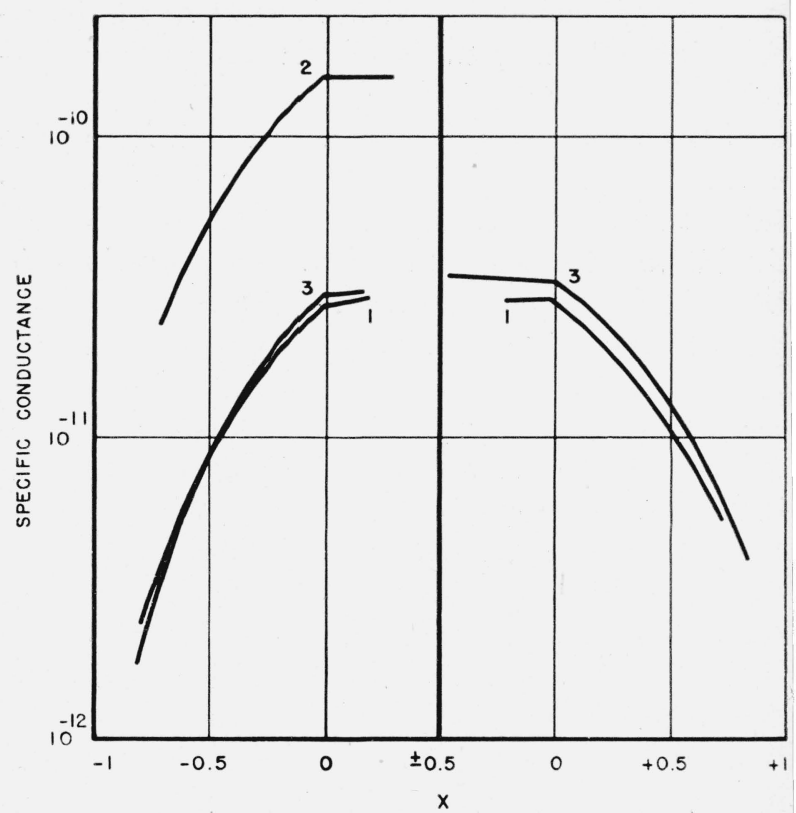

Figure 2.-Forward (left) and reverse (right) titration curves with picric acid and di-n-butylamine.

Curves: 1 , in benzene; 2 , in benzene containing 1 percent of methyl alcohol: 3 , in dioxane.

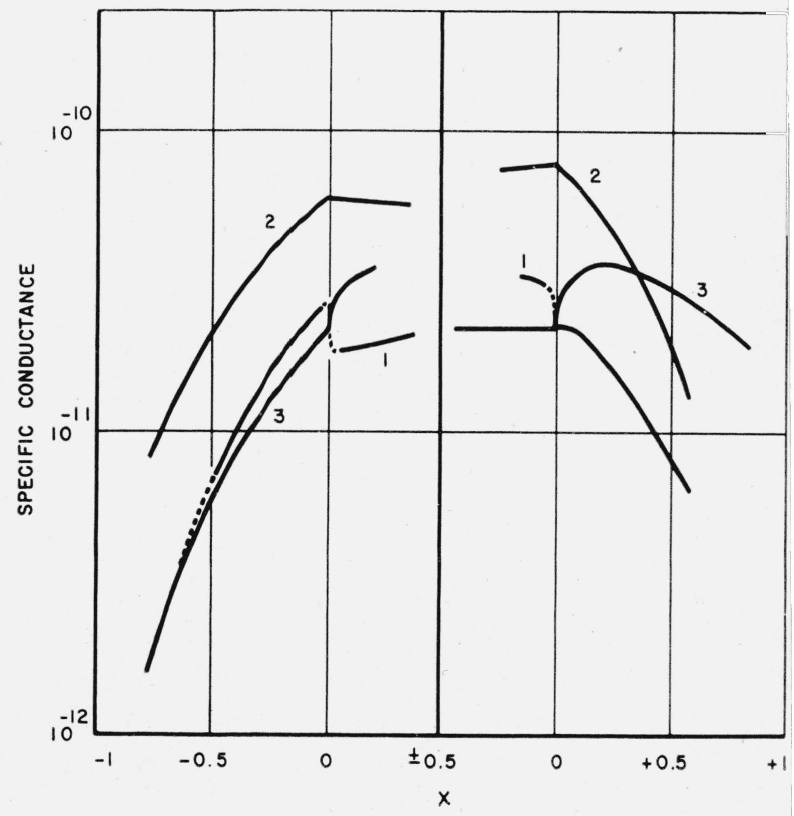

FiguRE 3.-Forward (left) and reverse (right) titration curves with picric acid and triethylamine.

Curves: 1 , in benzene; 2 , in benzene containing 1 percent of methyl alcohol; 3 , in dioxane. 


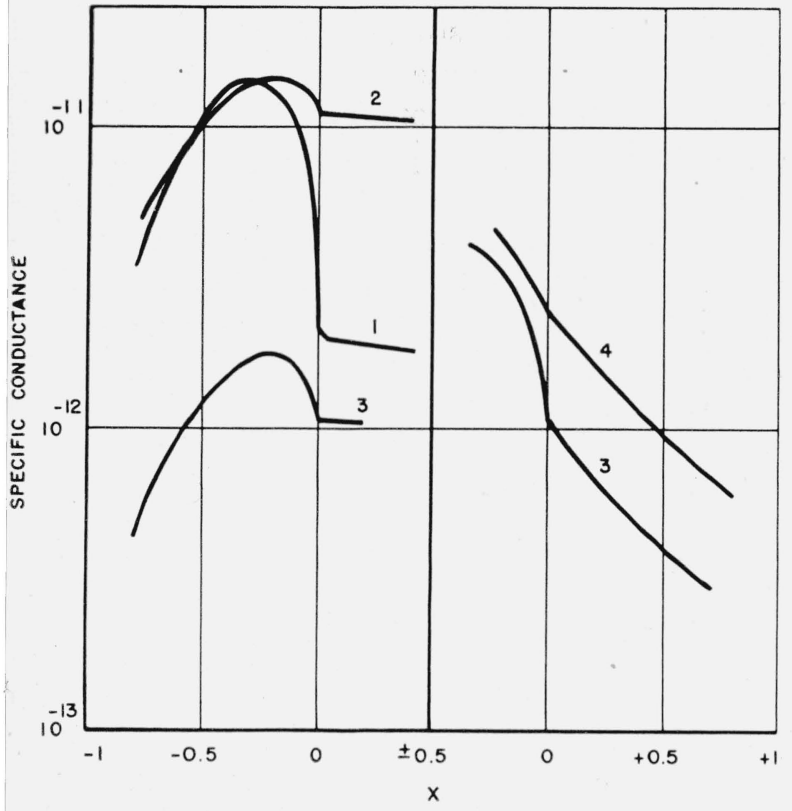

FiguRE 4.-Forward (left) and reverse (right) titration curves with trichloroacetic acid and triethylamine.

Curves: 1 , in benzene; 2 , in benzene containing 1 percent of methyl alcohol; 3 , in dioxane; 4 , in dioxane containing 1 percent of methyl alcohol.

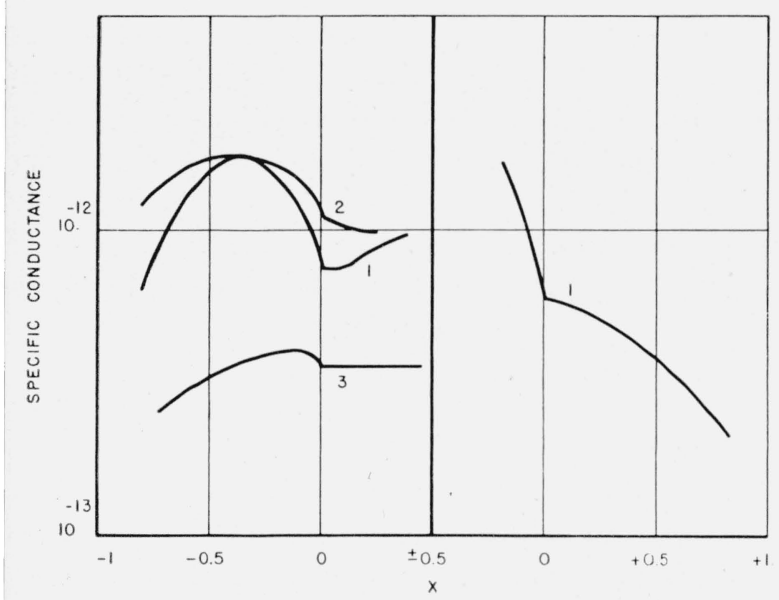

FIGURE 5.-Forward (left) and reverse (right) titration curves with trichloroacetic acid and di-n-butylamine.

Curves: 1 , in benzene; 2 , in benzene containing 1 percent of methyl alcohol; 3, in dioxane.

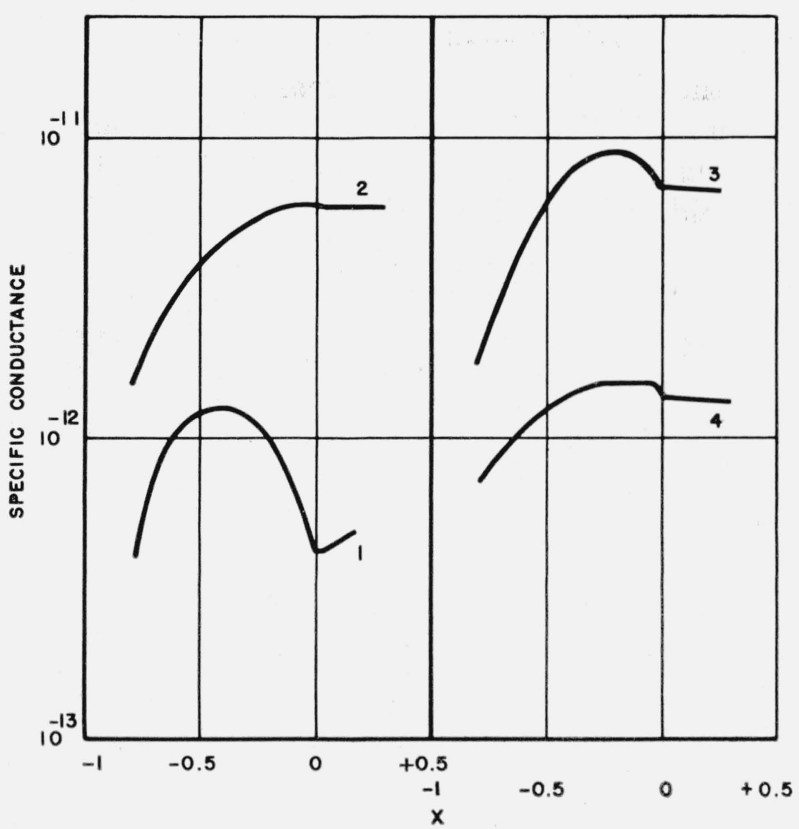

FigURE 6.-Forward titration curves using trichloroacetic acid (left) and camphorsulfonic acid (right).

Curves with trichloroacetic acid and $n$-heptylamine: 1 , in benzene; $[2$, in benzene containing 1 percent methyl alcohol. Curves using camphorsulfonic acid in dioxane: 3 , with triethylamine; 4 , with di- $n$-butylamine.

\section{Titrations With Picric Acid and Di-n-butylamine}

Curves representing the titrations with picric acid and di-n-butylamine are shown in figure 2 . Those numbered 1,2, and 3 are the titrations in benzene, in benzene containing 1 percent by volume of methyl alcohol, and in dioxane, respectively. Conductances of the initial solutions containing only free acid or base are not shown on the plots. These conductances were less than one-hundredth the conducance of the salt at the equivalence point. All curves, both for the forward and reverse titrations, have the same general shape and show a pronounced break at the equivalence point. The conductance increases continually until the equivalence point is reached and remains substantially constant thereafter. This is the typical behavior in cases where the conductance is largely determined by the concen- 
tration of salt formed during the neutralization, and the titrations bear a formal analogy to conductimetric titrations of weak acids and bases in aqueous media, where the extent of ionization of the acid and base is relatively small compared to that of the salt. A comparison of the forward titration curve with the corresponding curve for the reverse titration shows that they are nearly superimposable. Unlike many of the titrations to follow, there is no indication of any specific interaction between salt and free acid or base of such a nature as to influence markedly the conductance of these solutions. The conductances are not very different in the two solvents, benzene and dioxane, and the addition of the small amount of alcohol to the benzene has merely the expected effect of shifting the titration curve to higher values of conductance.

\section{Titrations With Picric Acid and Triethylamine}

When similar titrations, shown in figure 3 , are made with picric acid and triethylamine rather than di- $n$-butylamine, the results are quite different and dependent upon the solvent. Curves 1,2 , and 3 are again the titrations in benzene, in benzene containing 1 percent of methyl alcohol, and in dioxane, respectively. With the first additions of base during the forward titration in benzene, the conductance is unstable and tends to drift rapidly with time toward higher values. As the titration proceeds, these drifts become less pronounced and practically vanish after somewhat more than half of the acid is neutralized. The conductance then steadily increases in the expected manner until a second drift, this time toward decreasing conductance, occurs at or very close to the equivalence point. The reverse titration shows a corresponding drift toward increasing conductance in the immediate vicinity of the equivalence point, but none during the initial part of the titration. The regions of marked instability are indicated by the dotted portions of the curves.

From a comparison of the forward and reverse titrations it would appear that there are two quite different values representing the conductance of triethylammonium picrate. The conductance near the equivalence point when approached from the forward titration is about 20 percent greater than for the reverse titration, although the concentration of salt is about 10 percent greater in the latter case. To study this effect further and to ascertain which is the proper value for the con ductance of the salt, a solution of purified triethylammonium picrate, approximately 0.009 molar, was introduced into the cell. Upon the addition of one drop of the solution of picric acid, the conductance began to drift and continued until it reached a steady value in about 10 minutes, which was approximately 30 percent higher than the original. Subsequent small additions of acid did not produce any further drifts in conductance. On back titrating with the stock solution of triethylamine to give a drop excess, the conductance drifted back approximately to its original value. This change was not dependent upon the continual passage of current through the solution. It is evident from the above experiment and from the instability noticed during the initial part of the forward titration that this drift in conductance toward abnormally high values is associated with solutions containing both salt and free picric acid. The process is reversible and possibly suggests a slow attainment of some ionic equilibrium involving a reaction between the acid and the salt. The possibility that relatively slow reactions may occur in such solvents has been suggested by Dietz and Fuoss [4], who found that the conductances of mixtures of tributylammonium chloride and lead abietate in toluene drifted with time in a manner which indicated a slow ionic reaction between these two salts. The failure of larger excesses of picric acid to produce additional drifts indicates a saturation effect requiring only a very small concentration of acid.

Curves 2 in figure 3 represent titrations with the same solutions, the difference being that 1 percent by volume of methyl alcohol was added to the benzene before titrating. Unlike the titrations in pure benzene, no drifts in conductance were noted during any part of either the forward or reverse titrations. The shapes of both curves are similar and now resemble those obtained with picric acid and di-n-butylamine.

Still different behavior is shown by the titrations in dioxane. The conductance during the forward titration, instead of leveling off beyond the equivalence point, increases at a faster rate than before. In the reverse titration the conductance reaches a maximum value when only about 80 percent of the base has been neutralized and then decreases rather rapidly up to the equiva- 
lence point. Beyond this point the conductance remains practically constant. Conductances were stable throughout the titrations. In view of the relatively low conductance of the base, the conductance of solutions containing both salt and free base are unexpectedly high. The conductance at the maximum of the reverse titration is $3.7 \times 10^{-11}$ compared to a value of $1.4 \times 10^{-11}$ at the corresponding point in the forward titration where the concentration of salt is the same. There is evidently some rather strong interaction between the salt and base in dioxane of such a nature as to increase markedly the extent of ionization over that produced by the salt alone.

\section{Titrations With Trichloroacetic Acid}

Figures 4, 5, and the left side of figure 6, show the curves for titrations with trichloroacetic acid. All curves for the forward titrations are qualitatively similar. They rise to a maximum between half neutralization and the equivalence point. Immediately beyond the equivalence point, the conductance tends to level off so that breaks in the curves at the equivalence points are very pronounced. Curves for the forward and reverse titrations have entirely different shapes. The conductance increases throughout the reverse titration but at a faster rate beyond the equivalence point than before. The breaks, though not so striking as in the case of the forward titrations, are still quite definite.

It is apparent from these curves that solutions containing free acid as well as salt have abnormally high conductances. The outstanding illustration is the titration of trichloroacetic acid with triethylamine, curve 1 of figure 4 . The maximum occurs at $X=0.3$, which corresponds to a solution containing 0.0065 -molar salt and 0.0027 molar acid. The specific conductance of this solution is about $1.5 \times 10^{-11}$, whereas that at the equivalence point corresponding to a substanially higher salt concentration of 0.0090 molar is only $2.0 \times 10^{-12}$. The drop in conductance just before the equivalence point is extremely sharp. On titrating the last 5 percent of acid there is a threefold drop in conductance. This behavior suggests a specific reaction between acid and salt leading to much greater ionic dissociation than can be attributed to the ordinary dissociation of the salt itself.
In several instances, excess base causes an appreciable increase in conductance. For example, the forward titration curves for trichloroacetic acid with di- $n$-butylamine and $n$-heptylamine in benzene, curve 1 in figure 5 , and curve 1 in figure 6 , respectively, show a gradual rise in conductance beyond the equivalence point. Some interaction between salt and base is indicated, but the effect is relatively less important than in the case of acid and salt, and is insufficient to cause a maximum in the curve for the reverse titration.

The maxima, in the forward titrations are more pronounced in benzene than they are in dioxane or in benzene containing the small amount of alcohol. A comparison of the titrations of trichloroacetic acid with $n$-heptylamine in benzene and in benzene containing 1 percent of methyl alcohol, curves 1 and 2, respectively, in figure 6 , shows that addition of alcohol has nearly eliminated the maximum and all but obsecured the end point. The effect of adding alcohol to dioxane is shown for the reverse titrations with trichloroacetic acid and triethylamine. The titration in pure dioxane is represented by right-hand curve 3 in figure 4, and that after the alcohol had been added by curve 4 . In the latter case, the end point is much less pronounced, and it would probably not be evident at all upon the addition of somewhat larger amounts of alcohol.

\section{Titrations With Camphorsulfonic Ācid}

The very limited solubility of camphorsulfonic acid precluded its use in titrations in benzene at the desired concentration. Two titrations were made in dioxane, using triethylamine and di- $n$ butylamine, curves 3 and 4 in figure 6 , respectively. The appearance of maxima in these curves, just as in the titrations with trichloroacetic acid, shows a similarity in behavior of the two acids.

\section{Accuracy of End Points}

Except for the titrations with picric acid and triethylamine in pure benzene, the end points were determined by the intersection of smooth curves, usually linear, drawn through points immediately on each side of the break in the titration curve. Agreement with the equivalence points calculated from the independently determined concentrations of the stock solutions was within 3 percent in every case. The average deviation was somew hat 
less than 1 percent and within the experimental uncertainties. In the special case of titrations with picric acid and triethylamine in benzene, the end point was indicated by the previously described drift in conductance which was evidently confined to a region within 1 percent of the true equivalence point. Because of the nature of the end point, however, this particular titration is less suitable than the others; and it is apparently not applicable to certain other tertiary amines. Preliminary titrations with tributylamine or triamylamine, although qualitatively similar to those with triethylamine, gave a slower and less pronounced drift in conductance, which did not give a satisfactory indication of the end point. Although the effect of alcohol was not studied in these preliminary titrations, its addition would, in all probability, improve the titrations in the manner observed with triethylamine.

\section{Discussion}

It is apparent from the various graphs that the titrations, with the exception of those with picric acid and triethylamine in pure benzene, follow one of two general patterns. The simplest type includes all the titrations involving picric acid and di- $n$-butylamine, and those with picric acid and triethylamine in benzene containing the small amount of alcohol. Corresponding curves for the forward and reverse titrations have the same general shape, and the conductance is controlled almost entirely by the concentration of salt formed during the neutralization. For the second type, curves for the forward and reverse titrations have entirely different, though complementary, shapes. In all forward titrations employing trichloroacetic acid or camphorsulfonic acid, the conductance passes through a maximum well before the equivalence point is reached. This maximum occurs in the reverse titration in the particular case involving picric acid and triethylamine in dioxane. Except in the titrations with picric acid and triethylamine, the character of the curves did not depend upon the solvent used.

In order to account for the behavior of titrations of this second type, some specific reaction between salt and acid, or between salt and base in one instance, would seem to be required. ${ }^{3}$ Although independent data in dilute solutions are lacking, amine salts of carboxylic acids do form complexes with excess acid under certain conditions. Ralston [5] has obtained evidence from the freezing point diagram of the system, octadecylamine-acetic acid, of the presence not only of the 1:1 compound representing the simple salt but also of a compound containing two molecules of acid to one of base. Prideaux [6] found that the conductance of the two component system, diethylamine-propionic acid, when corrected for changes in viscosity had a maximum value in the neighborhood of 70 mole percent of acid, and concluded that a complex salt having a higher conductance than the simple salt was formed.

That carboxylic acids associate into dimers through the formation of hydrogen bonds in the vapor phase and in solvents like benzene is well established [7]. The reaction between simple salt and acid, formulated by $\mathrm{HB}^{+} . . \mathrm{A}^{-}+\mathrm{HA}=$ $\mathrm{HB}^{+}$. . $\mathrm{HA}_{2}{ }^{-}$, might be attributed to the formation of a complex anion having a similar structure except that only one hydrogen bond could be formed. For the case of trichloroacetic acid, this structure would be represented by

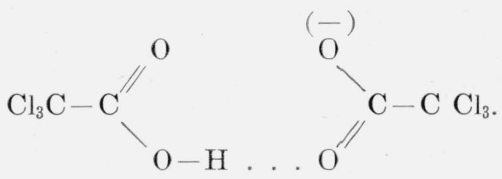

Formation of such a complex salt would be expected to enhance the conductance of the solution. The larger size of the complex anion and the better shielding of its charge should lead to a higher degree of dissociation of ion pairs into free ions, than for the simple salt, by diminishing the attractive forces between the oppositely charged ions.

It is interesting to note that the reaction between acid and salt still takes place when dioxane rather than benzene is the slovent. Dioxane, like other solvents the molecules of which contain acceptor atoms, tends to destroy hydrogen bonds

\footnotetext{
3 The high conductances of solutions containing salt and acid together were noted by La Mer and Downes in the titration of trichloroacetic acid with diethylamine in benzene, previously mentioned [1]. These authors concluded that there was a Debye salt effect which increased greatly the ionic dissociation of the acid. However, the ionic strengths of these solutions, as evidenced by their extremely low conductances, are so small that such an interpretation would hardly seem valid.
} 
between other molecules because of its own tendency to form hydrogen bonds with them. Carboxylic acids, for example, do not associate into dimers in dioxane. Consequently, a complex anion of the type postulated above would hardly be expected to be sufficiently stable in dioxane to give the observed maxima in the titration curves, unless an exceptionally stable structure resulted. Although the presence of a negative charge on the complex ion should tend to strengthen this conventional type of hydrogen bonding, it would appear more likely that the ion has a somewhat different structure. Resonance in the carboxylate ion gives both carbon-oxygen bonds a partial double bond character and makes them equivalent. In the complex anion the proton of the second carboxyl group could assume a position where it was equally shared by all four oxygen atoms as indicated by the structure

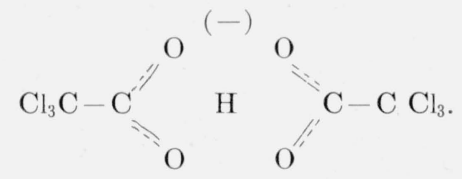

Rather than the planar configuration shown, the four oxygens would, in all probability, be located tetrahedrally around the proton. Because of the compact structure and the gain in energy through complete resonance of both carboxyl groups, this configuration should be more stable than the previous structure involving the more conventional type of hydrogen bond.

The similarity in behavior of trichloroacetic acid and camphorsulfonic acid indicates a corresponding interaction between the latter acid and its salt. A bonding of the sulfonic acid group with the sulfonate ion in a manner analogous to that formulated in the case of the carboxylic acid would appear likely.

The behavior of the titrations involving picric acid and triethylamine in dioxane, where solutions containing both salt and base have unexpectedly high conductances, suggests a strong reaction between salt and base leading to the formation of a hydrogen-bonded complex cation, as $\mathrm{R}_{3} \mathrm{~N}^{+}-\mathrm{H}$ ... $\mathrm{NR}_{3}$ in this particular case. The possibility of forming this same complex cation exists in other cases, for example, in the titrations of trichloroacetic acid or camphorsulfonic acid with triethylamine in dioxane. Its actual existence to any significant extent is ruled out by the experimental data which fail to show any marked rise in conductance during the forward titrations after the equivalence point has been reached. This fact is not too surprising as the salts exist almost entirely in the form of ion pairs so that the behavior of the cation is influenced by the nature of the attached anion.

The anomalous behavior of titrations with picric acid and triethylamine in benzene has been discussed previously. In a few of the other titrations, small variations in conductance with time took place which were not confined to any particular portion of the titration curves. Since a direct current bridge was employed, it is quite possible that these variations were associated with some polarization phenomenon and could be eliminated by an alternating current method. These drifts were sometimes toward lower and sometimes toward higher conductances. In the titrations with picric acid and di- $n$-butylamine in benzene, for example, drifts toward lower conductance, or higher resistance, were noted. The conductance immediately after addition of the titrating solution and mixing was several percent higher than the steady value reached in 10 or 15 seconds. Reversal of the polarity of the bridge caused an immediate increase in conductance to or slightly above the initially observed value which was then followed by a decrease to the same steady value as before. Subsequent mixing also tended to restore the original value. A rather detailed study of an apparently analogous effect also with direct current, has been reported by Fuoss and Elliott [8] in which the electrolyte was tri-nbutylammonium picrate and the solvent, tricresyl phosphate, a highly viscous medium with a relatively low dielectric constant. The attainment of a steady state required a much longer time and the change in conductance was much greater in this case. These authors attributed this slow increase in effective resistance of the development of a space charge in the solution near the electrodes which deceased the potential gradient across the main body of the solution.

Drifts toward higher conductances, observed for example in titrations with trichloroacetic acid and $n$-heptylamine in dioxane, occurred more slowly and several minutes were required to reach a steady state. As before, mixing the solution restored the initial value. This drift cannot be attributed to the development of a space charge. 
On reversal of the polarity, however, an effect similar to that described in connection with the titrations involving picric acid and di- $n$-butylamine was also observed, so that there would appear to be a superposition of at least two different effects. Observation of these drifts was more or less incidental since they did not interfere with the titrations, provided a consistent procedure was followed in taking the readings. Where the drifts were relatively slow, the initial conductances, reproducible on repeated mixing, were used. The final or steady values were used where the drift was quite rapid. In a majority of cases the systems were quite stable and no variation in conductance was observed either on reversal of the polarity or on mixing. Where drifts were observed, addition of the small amount of alcohol to the solvent eliminated them. To explain these drifts in certain systems and their absence in others will require a more thorough investigation employing both alternating and direct current methods.

The results of the present investigation show that conductimetric titrations of acids and bases are feasible in solvents of the lowest dielectric constants even though the conductances are lower by a factor of $10^{-8}$ than they would be in water. The diversified behavior of these systems, not anticipated from studies in the usual solvents, makes generalizations difficult. Qualitatively, the assumption of the formation of a complexion accounts for the behavior of a number of the titrations, but additional experimental data will be required before a more complete interpretation in terms of reaction equilibria can be made. The data also are of interest in connection with the general problem of acid-base equilibria in media of low dielectric constant. While in certain instances such reactions do seem to follow a simple mass law [9], in others the behavior is more complicated [10]. In addition to factors such as association of the acids and salts, the formation of complex salts as suggested by the present studies may introduce additional complications, particularly in cases where carboxylic or sulfonic acids are employed.

\section{References}

[1] V. K. La Mer and H. C. Downes, J. Am. Chem. Soc. 53, 888 (1931).

[2] P. Walden, Z. physik. Chem. 14\%, 1 (1930).

[3] C. A. Kraus, J. Franklin Inst. 225, 687 (1938).

[4] V. Dietz and R. M. Fuoss, J. Am. Chem. Soc. 60, 2394 (1938)

[5] W. O. Pool, H. J. Harwood, and A. W. Ralston, J. Am. Chem. Soc. 6\%, 775 (1945).

[6] R. N. Coleman and E. B. R. Prideaux, J. Chem. Soc. 193\%, 1022.

[7] J. Karle and L. O. Brockway, J. Am. Chem. Soc. 66, 574 (1944).

[8] R. M. Fuoss and M. A. Elliott, J. Am. Chem. Soc. 67, 1939 (1945).

[9] Marion Maclean Davis and P. J. Schuhmann, unpublished data, National Bureau of Standards.

[10] V. K. La Mer and H. C. Downes, Chem. Rev. 13, 47 (1933).

Washington, February 12, 1947. 